\title{
PERFECT CATEGORIES
}

\author{
by JOHN ISBELL \\ (Received 16th December 1974)
}

\section{Introduction}

This note extends to categories Fountain's theorem (2) that for a perfect monoid $S$, every flat $S$-set is projective. (The converse is known (4).)

Fountain used the theorem for monoids to prove that perfection is also equivalent to the pair of properties:

$A$. Every locally cyclic $S$-set is cyclic.

$M_{R}$. The principal right ideals of $S$ satisfy the minimum condition.

A similar result for categories $\mathscr{C}$ is a corollary, since it was known (4) that $\mathscr{C}$ is perfect if and only if it has property $A$ and every monoid $\mathscr{C}(X, X)$ is perfect.

Theorem. The following three conditions on a category $\mathscr{C}$ are equivalent:

(a) $\mathscr{C}$ is perfect;

(b) Every flat (set-valued) functor on $\mathscr{C}$ is projective;

(c) Every weakly flat (set-valued) functor on $\mathscr{C}$ is projective.

The implication $(c) \Rightarrow(b)$ is trivial; $(b) \Rightarrow(a)$ is known $(4)$; so we need to prove $(a) \Rightarrow(c)$.

\section{Definitions and background}

The basic reference for flat functors is the seminar notes of Grothendieck and Verdier (3), which treat them as generalised representable functors and call them " ind-objets" (" objet" meaning a representable functor). They are defined (3) as the direct limits of directed systems of representable functors. The standard term is now flat functor, although as far as I know it has not made its way from lectures into print. As with (flat) modules, so with functors $F: \mathscr{C} \rightarrow \mathscr{S}$, the property is equivalent to this: the set-valued functor ()$\otimes F$, on $\operatorname{cat}\left(\mathscr{C}^{\mathrm{op}}, \mathscr{S}\right)$ to $\mathscr{S}$, is left exact. There are results like that in (3), from which this result became clear when the tensor product $G \otimes F$ of contravariant $G$ and covariant $F$ was defined. F. W. Lawvere tells me that this was well known in Zurich certainly by 1966 . The explicit definition of $\otimes$ is simple enough (using the notation of (4); Latin letters denote points of $F$, which are ordered pairs consisting of an object $X$ of $\mathscr{C}$ and an element $e$ of $F(X)$, Greek letters denote morphisms of $\mathscr{C}$, and of course a juxtaposition $\alpha p$ is meaningful only when the object of $p$ is the domain of $\alpha$ ); form $G \otimes F$ from the coproduct 
of all $G(X) \otimes F(X)$ by identifying $(q \alpha, p)$ with $(q, \alpha p)$, for every meaningful instance of these expressions.

The definition of $\otimes$ was published by B. Mitchell (5), whose results do not touch on flatness but include these fundamentals: for representable $G=h_{y}$, $G \otimes F$ is $F(Y)$; for representable $F=h^{X}, G \otimes F$ is $G(X)$; in general, $\otimes$ is cocontinuous in each variable.

Weakly flat functors (as in Stenström (5)) are the direct limits of projective functors. Recall that projective functors are the retracts of free functors; in turn, free functors are coproducts of representable ones. One verifies by inspection that each of these constructions preserves the following property which the representable functors plainly have:

$\left.{ }^{*}\right)$ In $F$ there are no relations $\alpha p=\beta q$ except those given by relations $\alpha v=\beta \delta$ in $\mathscr{C}$ and points $o$, by means of $p=v o, q=\delta o$; and there are no relations $\alpha p=\beta p$ except those given by $\alpha v=\beta v, p=v o$.

Thus $\left(^{*}\right)$ is true of weakly flat functors $F$. (Actually it characterises them. Grothendieck-Verdier showed (3, Theorem 8.3.3) that this and indecomposability characterise flat functors.)

Proof of $(a) \Rightarrow(c)$

We can use Fountain's lemmas to prove:

Theorem. If $\mathscr{C}$ is perfect then every weakly flat functor $F: \mathscr{C} \rightarrow S$ is projective.

Proof. Perfection implies (4) property $A$ : every locally cyclic functor is cyclic, or in other words every ascending chain of cyclic subfunctors is finite. Then (following Fountain's numbering of lemmas):

(2) Every generating set of points of $F$ contains an irredundant generating set.

For let $M$ be the set of all maximal cyclic subfunctors $\mathscr{C} p$ of $F$. A generating set $S$ must include, for each $\mathscr{C} p$ in $M$, some $s$ such that $p \in \mathscr{C} s$; then

$$
\mathscr{C} p \subset \mathscr{C} s, \mathscr{C} p=\mathscr{C} s \in M \text {. }
$$

(3) The indecomposable summands of $F$ are cyclic.

For let $S$ be an irredundant generating set. The claim is that $\mathscr{C} p, \mathscr{C} q$ are disjoint for $p \neq q$ in $S$. Indeed, if not, then $\left(^{*}\right)$ gives $p=v o, q=\delta o$, with $\mathscr{C} o$ containing $\mathscr{C} p$ and $\mathscr{C} q$, contrary to maximality.

The indecomposable summands are, of course, also flat (but we need only $\left.{ }^{*}\right)$, which they evidently inherit).

(5) The indecomposable summands of $F$ are projective.

For this we must recall that perfection implies (4) property $D$ : every isotropy set has a minimal left ideal generated by an idempotent. (An isotropy 
set, meaning a coset of an identity $1_{X}$ in a left congruence, is (4) simply an isotropy set in $\mathscr{C}(X, X)$, or left unitary subsemigroup (2).)

Let $E$ be an indecomposable summand of $F$. $E$ being flat cyclic, of the form $h^{X} / \rho$ for some left congruence $\rho$, we shall construct an isomorphism with a projective $h^{X} \alpha$, where $\alpha: X \rightarrow X$ is idempotent. $\left(h^{X} \alpha(Y)\right.$ is the subset of $h^{x}(Y)$ consisting of all elements $\xi \alpha ; h^{X}$ retracts onto $h^{x} \alpha$ by $\phi$, where $\phi_{Y}(\xi)=\xi \alpha$.) We need to note that when $\beta \rho=v \rho$ in $E(Y)$, there exists $\theta: X \rightarrow X$ in $\mathscr{C}, \rho$ equivalent to $1_{X}$, such that $\beta \theta=v \theta$. For $\beta\left(1_{X} \rho\right)=v\left(1_{X} \rho\right)$ and $\left(^{*}\right)$ yield $\beta \delta=\nu \delta$ and $1_{X} \rho=\delta o$ for some $\delta: W \rightarrow X$ and $o \in E(W)$. But $1_{X} \rho$ generates $E$, so $o$ is $\eta\left(1_{x} \rho\right)$ and we may put $\theta=\delta \eta$.

Let $B=1_{x} \rho$ and let $B \alpha$ be a minimal left ideal of $B$ generated by idempotent $\alpha$. By Lemma 8.12 of (1), $\alpha B$ is a minimal right ideal of $B$. By the remark above, for each $\beta$ in $B$ (since $\beta \rho=\alpha \rho$ ) there is $\theta$ in $B$ with $\beta \theta=\alpha \theta$. So $\beta \theta B \subset \alpha B, \alpha B=\beta \theta B$ by minimality, and therefore $\alpha \in \beta B$.

Define $f: h^{x} / \rho \rightarrow h^{x} \alpha$ by $f_{Y}(\xi \rho)=\xi \alpha$. If $\xi \rho$ is equal to $\zeta \rho$, then $\xi \beta=\zeta \beta$ for some $\beta$ in $B$ (by the remark above), so $\xi \alpha=\zeta \alpha$ since $\alpha \in \beta B$. Evidently $f$ is natural, and surjective. Since $(\xi \alpha) \rho=\xi(\alpha \rho)=\xi\left(1_{x} \rho\right)=\xi \rho, f$ is injective and (5) is proved.

The theorem follows since $F$ is a coproduct of projectives.

\section{REFERENCES}

(1) A. H. Clifford and G. B. Preston, The algebraic theory of semigroups, Vol. II (Math. Surveys no. 7, Amer. Math. Soc., 1967). 87-93.

(2) J. Fountain, Perfect semigroups. Proc. Edinburgh Math. Soc. 20 (1976),

(3) A. Grothendieck et J. L. Verdier, Prefaiscealix (Lecture Notes in Mathematics 269, Springer-Verlag, 1972), 1-217.

(4) J. R. IsBelL, Perfect monoids, Semigroup Forum 2 (1971), 95-118.

(5) B. Mitchell, The dominion of Isbell, Trans. Amer. Math. Soc. 167 (1972), 319-331.

(6) B. Stenström, Flatness and localization over monoids, Math. Nachr. 48 (1971), 315-334.

State University of New York at Buffalo

AMHERST

NEW YORK 14226

E.M.S. $-20 / 2-G$ 